\title{
Neutrinos as cosmic messengers
}

\author{
J. W. F. Valle \\ Instituto de Física Corpuscular, C.S.I.C. - Universitat de València \\ Edificio de Institutos de Paterna, Apartado 22085, E-46071 València, Spain
}

\begin{abstract}
.
I briefly review the current status of neutrino oscillation parameters and discuss the role of neutrinos as cosmological probes, that could possibly induce the baryon asymmetry as well as the dark matter in the Universe. I comment on the origin of neutrino masses in seesaw-type and low-scale models and mention some of their laboratory signals.
\end{abstract}

\section{STATUS OF NEUTRINO OSCILLATIONS}

The discovery of neutrino oscillations marks a turning point in particle physics as it implies that neutrinos have masses. Given their weak interaction, neutrinos play a special role as cosmic probes as they may provide information about very early stages of the evolution of the Universe. Last, but not least, understanding their properties may provide a valuable clue of what may lie ahead of the Standard Model (SM) of basic interactions.

Here I summarize the status of the neutrino oscillation results after the Neutrino 2008 conference [1], given in Ref. [2]. Evidence for neutrino oscillations coming from "celestial" (solar and atmospheric) neutrinos is unambiguously confirmed by "laboratory" neutrinos produced at reactors and accelerators. The basic theoretical layout for the description of neutrino oscillation data has been given almost thirty years ago and involves the concept of the lepton mixing matrix, the lepton analogue of the quark mixing matrix. In its simplest unitary 3-dimensional form is given as [3]

$$
K=\omega_{23} \omega_{13} \omega_{12}
$$

where each $\omega$ is effectively $2 \times 2$, characterized by an angle and a basic Majorana CP phase present already with two generations of neutrinos [3]. These do not affect oscillations [4], moreover, current neutrino oscillation data have no sensitivity to the remaining Dirac CP violation phase. Thus we set all three phases to zero. In this approximation oscillations depend on the three mixing parameters $\sin ^{2} \theta_{12}, \sin ^{2} \theta_{23}, \sin ^{2} \theta_{13}$ and on the two mass-squared splittings $\Delta m_{21}^{2} \equiv \Delta m_{21}^{2} \equiv m_{2}^{2}-m_{1}^{2}$ and $\Delta m_{31}^{2} \equiv \Delta m_{31}^{2} \equiv m_{3}^{2}-m_{1}^{2}$ characterizing solar and atmospheric transitions. The hierarchy $\Delta m_{21}^{2} \ll \Delta m_{31}^{2}$ implies that, to a good approximation, one can set $\Delta m_{21}^{2}=0$ in the analysis of atmospheric and accelerator data, and $\Delta m_{31}^{2}$ to infinity in the analysis of solar and reactor data.

The analysis of the data requires accurate calculations of solar and atmospheric fluxes, neutrino cross sections and response functions, as well as a careful description of neutrino propagation in the Sun and the Earth, taking into account matter effects. 

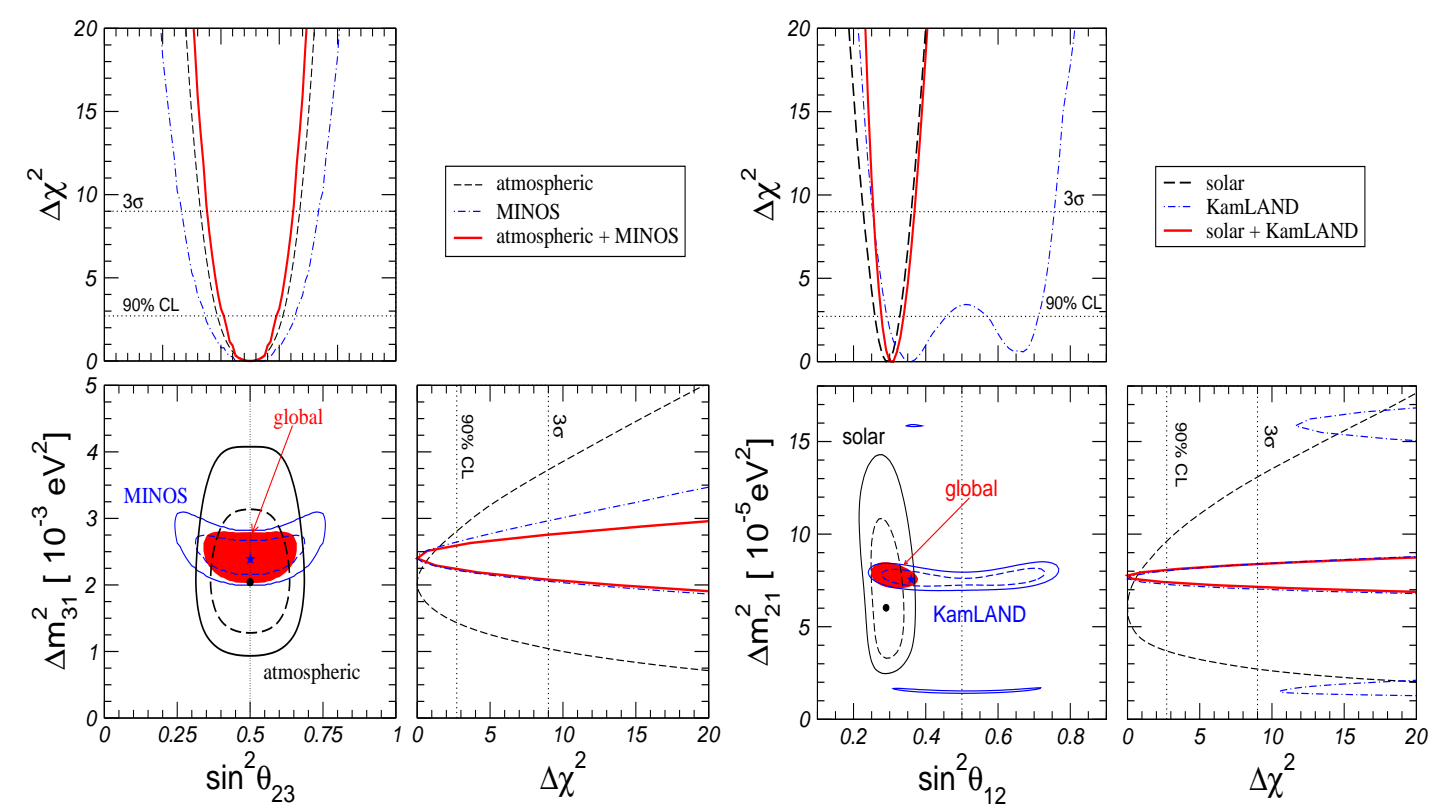

FIGURE 1. Neutrino oscillation parameter regions after Neutrino 2008 conference, from Ref. [2].

The resulting three-neutrino oscillation parameters obtained in the global analysis are summarized in Figs. 1 and 2 The analysis includes all new neutrino oscillation data, as of the recent Neutrino 2008 conference [2]. These include the data released this summer by the MINOS collaboration, the data of the neutral current counter phase of the SNO solar neutrino experiment, as well as the latest KamLAND and Borexino data.

The left panel gives the leading "atmospheric" oscillation parameters $\theta_{23} \& \Delta m_{31}^{2}$ from the interplay of data from artificial and natural neutrino sources. We show $\chi^{2}$ profiles and allowed regions at $90 \%$ and $99.73 \%$ CL ( 2 dof) for atmospheric and MINOS, as well as the $99.73 \% \mathrm{CL}$ region for the combined analysis (including also K2K). The dot, star and diamond indicate the best fit points of atmospheric data, MINOS and global data, respectively. We minimize with respect to $\Delta m_{21}^{2}, \theta_{12}$ and $\theta_{13}$, including always solar, KamLAND, and CHOOZ data.

The right panel gives the corresponding "solar" oscillation parameters $\theta_{12} \& \Delta m_{21}^{2}$ obtained by combining solar and reactor neutrino data. We show $\chi^{2}$-profiles and allowed regions at $90 \%$ and $99.73 \%$ CL ( 2 dof) for solar and KamLAND, as well as the 99.73\% CL region for the combined analysis. The dot, star and diamond indicate the best fit points of solar data, KamLAND and global data, respectively. We minimize with respect to $\Delta m_{31}^{2}, \theta_{23}$ and $\theta_{13}$, including always atmospheric, MINOS, K2K and CHOOZ data.

The angle $\theta_{13}$ holds the key for future searches for $\mathrm{CP}$ violation in neutrino oscillations. Fig. 2 summarizes the information on $\theta_{13}$ from present data, the right panel compares the situation in 2007 and 2008. One sees that the current data slightly prefer a nonzero value for $\theta_{13}$, though this is not significant and we interpret this as giving a bound on $\theta_{13}$. An important contribution to this bound comes, of course, from the CHOOZ reactor experiment combined with the determination of $\left|\Delta m_{31}^{2}\right|$ from at- 

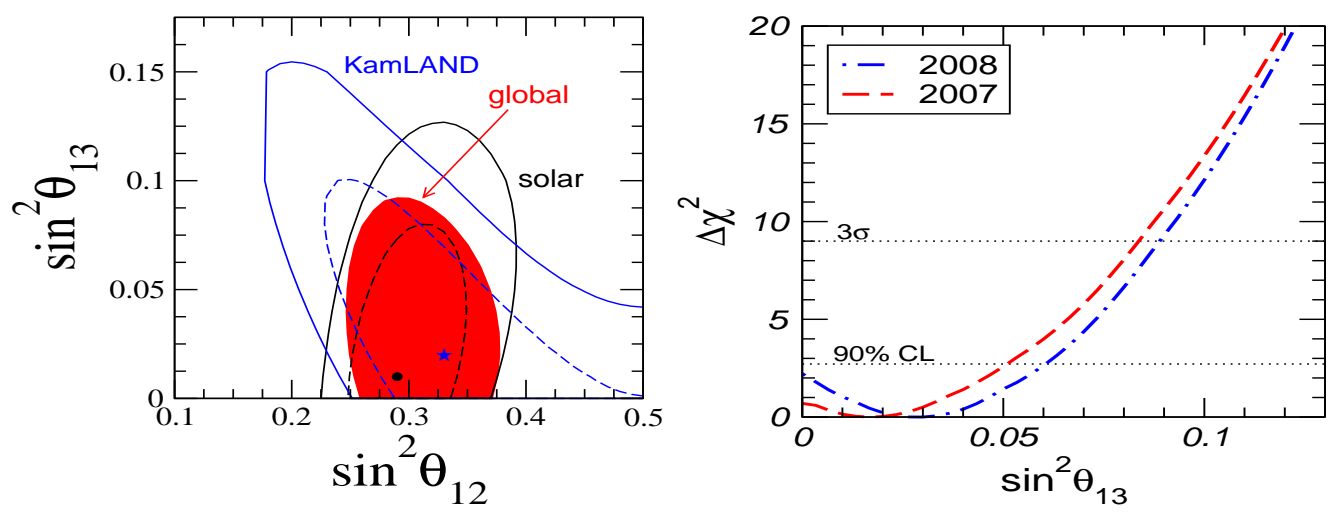

FIGURE 2. Constraints on $\sin ^{2} \theta_{13}$ from different parts of the global data given in Ref. [2].

mospheric and long-baseline experiments. The complementarity of different data sets provides a non-trivial constraint on $\theta_{13}$, namely 1 :

$$
\sin ^{2} \theta_{13} \leq \begin{cases}0.060(0.089) & (\text { solar+KamLAND }) \\ 0.027(0.058) & (\text { CHOOZ+atm+K2K+MINOS }) \\ 0.035(0.056) & (\text { global data })\end{cases}
$$

Within a three-neutrino scheme CP violation disappears when two neutrinos become degenerate or when one of the angles vanishes [5]. As a result $\mathrm{CP}$ violation is doubly suppressed, first by the small ratio $\alpha \equiv \Delta m_{21}^{2} / \Delta m_{31}^{2}$ of the two mass-squared differences, and also by the small value of $\theta_{13}$. There is now an ambitious long-term effort towards probing $\mathrm{CP}$ violation in neutrino oscillations in long-baseline experiments [6, 7]. The current status of the determination of the parameter $\alpha$ is

$$
\alpha \equiv \frac{\Delta m_{21}^{2}}{\left|\Delta m_{31}^{2}\right|}=0.032, \quad 0.027 \leq \alpha \leq 0.038 \quad(3 \sigma),
$$

The growing precision of oscillation experiments also opens good prospects for probing small effects beyond Eq. (1) such as unitarity violation and other forms of non-standard neutrino interactions [3]. Here I wish to stress that reactor neutrino data play a crucial role in testing the robustness of solar oscillations vis a vis astrophysical uncertainties, such as magnetic fields in the solar radiative [8, 9, 10] or convective zone [11, 12, 13], leading to stringent limits on neutrino magnetic transition moments [14]. KamLAND has also played a key role in identifying oscillations as "the" solution to the solar neutrino problem [15] and also in pinning down the large-mixingangle oscillation solution among the previous wide range of possibilities [16].

However, there is still an ambiguity left in the interpretation of the solar data in the presence of non-standard neutrino interactions (NSI), illustrated in Fig. 3. Indeed, most neutrino mass generation mechanisms imply the existence of such dimension-6 opera-

\footnotetext{
1 Note: the bounds in Eq. (2) are given for 1 dof, while the regions in Fig. 2 (left) are 90\% CL for 2 dof
} 


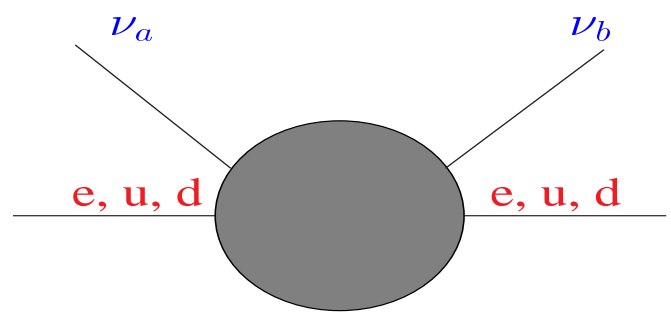

FIGURE 3. Non-standard neutrino interactions arise, e. g., from the non-unitary structure of charged current weak interactions characterizing seesaw-type schemes [3].

tors, typically sub-weak strength $\left(\sim \varepsilon G_{F}\right)$. They can be of two types: flavour-changing (FC) and non-universal (NU). Their presence leads to the possibility of resonant neutrino conversions even in the absence of neutrino masses [17]. For example, in the inverse seesaw model [18, 19] the non-unitary piece of the lepton mixing matrix can be sizeable, hence the induced non-standard interactions. Relatively sizable NSI strengths may also be induced in supersymmetric unified models [20] and models with radiatively induced neutrino masses [21, 22].

Although first determinations of atmospheric neutrino data allowed for an NSI interpretation [23], thanks to the large currently available statistics of data over a wide energy range the determination of $\Delta m_{31}^{2}$ and $\sin ^{2} \theta_{\text {ATM }}$ is now hardly affected by the presence of NSI, at least within the 2-neutrino approximation [24]. Future neutrino factories will substantially improve this bound [25].

In contrast, the determination of solar neutrino parameters is not yet robust against the existence of NSI [26], even if reactor data are included. One can show that even a small residual non-standard interaction may have dramatic consequences for the sensitivity to $\theta_{13}$ at a neutrino factory [27]. Improving the sensitivities on NSI constitutes a necessary step and opens a window of opportunity for neutrino physics in the precision age.

\section{LEPTON NUMBER VIOLATION}

Neutrino oscillations are blind to whether neutrinos are Dirac or Majorana fermions. In contrast, lepton number violating (LNV) processes, such as $0 v \beta \beta$ [28] 2, do have the potential of probing the intrinsic nature of neutrinos. For example, it will in general be sensitive to $\mathrm{CP}$ violation induced by the so-called Majorana phases [3, 4], inaccessible in conventional oscillations. Hence the search for neutrinoless double beta decay [33] constitutes a major goal for the future. It has also been argued that, in a gauge theory, irrespective of the mechanism that induces $0 v \beta \beta$, it necessarily implies a Majorana neutrino mass [28], as illustrated in Fig. 4. Indeed, in this resides the basic theoretical significance of $0 v \beta \beta$. This is known as the "black-box" theorem [28]. Although the theorem itself holds in any "natural" gauge theory, its quantitative implications are very model-dependent, for a recent discussion see [34].

\footnotetext{
${ }^{2}$ Neutrino transition magnetic moments [29, 30, 31, 32] provide another example of LNV processes.
} 


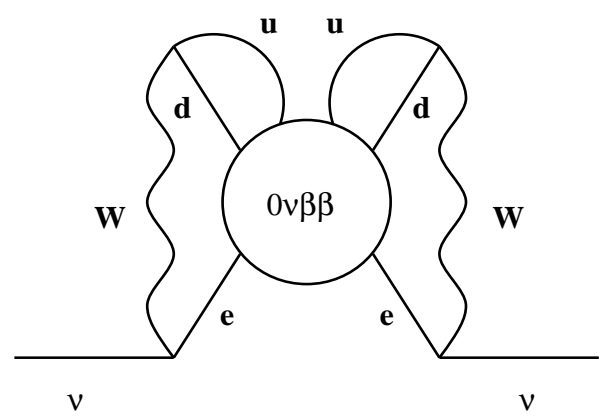

FIGURE 4. Neutrinoless double beta decay and Majorana mass are equivalent [28].

The observation of neutrino oscillations implies that $0 v \beta \beta$ must be induced by the exchange of light Majorana neutrinos, through the so-called mass-mechanism. The corresponding amplitude is sensitive both to the Majorana $\mathrm{CP}$ phases [3] , and also to the absolute scale of neutrino mass, neither of which can be probed in oscillations. Note that the absolute mass scale parameter probed by neutrinoless double beta decay is complementary to those probed in high sensitivity beta decay studies [35], and observations of the cosmic microwave background and large scale structure [36].

Taking into account current neutrino oscillation parameters [2] and state-of-the-art nuclear matrix elements [37] one can determine the average mass parameter $\left\langle m_{v}\right\rangle$ characterizing the neutrino exchange contribution to $0 v \beta \beta$, as shown in Fig. 10 of Ref. [38]. Models with quasi-degenerate neutrinos [39] [40] [41] give the largest $0 v \beta \beta$ signal. In normal hierarchy models there is in general no lower bound on $\left\langle m_{v}\right\rangle$, since there can be a destructive interference amongst the neutrino amplitudes (for an exception, see Ref. [42]; in that specific model a lower bound on $\left\langle m_{v}\right\rangle$ exists, which depends, as expected, on the value of the Majorana $\mathrm{CP}$ violating phase $\phi_{1}$ ). In contrast, the inverted neutrino mass hierarchy implies a "lower" bound for the $0 v \beta \beta$ amplitude.

The best current limit on $\left\langle m_{v}\right\rangle$ comes from the Heidelberg-Moscow experiment. There is also a claim made in Ref. [43] (see also [44]) which will be important to confirm or refute in future experiments. GERDA will provide an independent check of this claim [45]. SuperNEMO, CUORE, EXO, MAJORANA and possibly other experiments will further extend the sensitivity of current $0 v \beta \beta$ searches [46].

\section{LEPTON FLAVOR VIOLATION}

Given that neutrinos and charged leptons sit in the same electroweak doublet, and lepton flavour violation has been shown to occur in neutrino propagation, it is natural to expect that it may also show up as transitions directly involving the charged leptons themselves. Indeed, this occurs in seesaw-type schemes of neutrino mass, either through neutral heavy lepton exchange [47, 48, 49] or via supersymmetric contributions [50, 51, 52, 53]. Moreover, supersymmetry brings in the possibility of direct lepton flavour violation in the production of supersymmetric particles at the LHC [54].

As illustrated in Fig. 5 supersymmetry can lead to sizeable rates for lepton flavour 

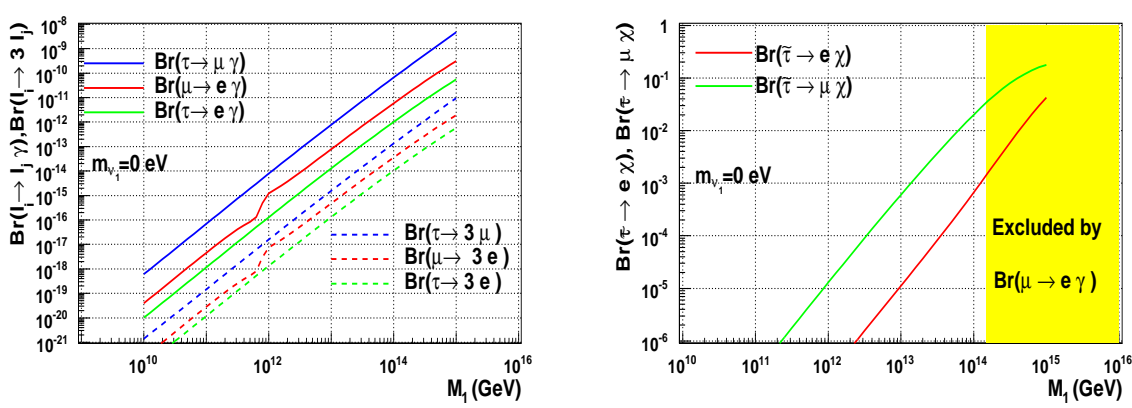

FIGURE 5. The left panel gives minimal type-I seesaw expectations for loop-induced LFV rates, versus the right-handed neutrino mass, while the right panel illustrates the possible direct LFV in the decays of staus at the LHC, details in Ref. [54].

violation processes even within the simplest minimal supergravity version of the typeI seesaw mechanism [54]. The figures illustrate the theoretical branching ratios for the LFV scalar tau decays, $\tilde{\tau}_{2} \rightarrow(e, \mu)+\chi_{1}^{0}$, as well as loop-induced LFV decays at low energy, such as $l_{i} \rightarrow l_{j}+\gamma$ and $l_{i} \rightarrow 3 l_{j}$, for given choice of the unknown seesaw parameters, see Ref. [54] for details. One can show that in some simple scenarios for the unknown right-handed parameters, the ratios of LFV branching ratios correlate with neutrino oscillation parameters. If the overall mass scale of the left neutrinos and the value of the reactor angle were known, the study of LFV allows, in principle, to extract information about the so far unknown right-handed neutrino parameters.

It is remarkable that, in general, the rates for lepton flavour violation processes may be sizeable, despite the small values of the light neutrino masses determined in current neutrino experiments. Indeed, an important theoretical point is that lepton flavour violation and $\mathrm{CP}$ violation can occur in the massless neutrino limit [47, 55, 56, 48]. As a result the allowed rates are not suppressed by the smallness of neutrino masses. In the extended seesaw scheme one can understand the interplay of both types of contributions. It is shown [19] that $\operatorname{Br}(\mu \rightarrow e \gamma)$ and the nuclear $\mu^{-}-e^{-}$conversion rates lie within planned sensitivities of future experiments such as PRISM [57]. The quasi-Dirac neutral heavy leptons present in such extended seesaw models may mediate large LFV even in the absence of supersymmetry and,if they have masses around $\mathrm{TeV}$ or so, may be directly produced at accelerators [58].

\section{THEORY OF NEUTRINO MASS}

Despite the great experimental progress neutrino physics has recently undergone, the ultimate origin of neutrino mass remains one of the most well kept secrets of nature. Here I will not dwell upon the various options to endow neutrinos with mass, however I will mention the main broad options. 

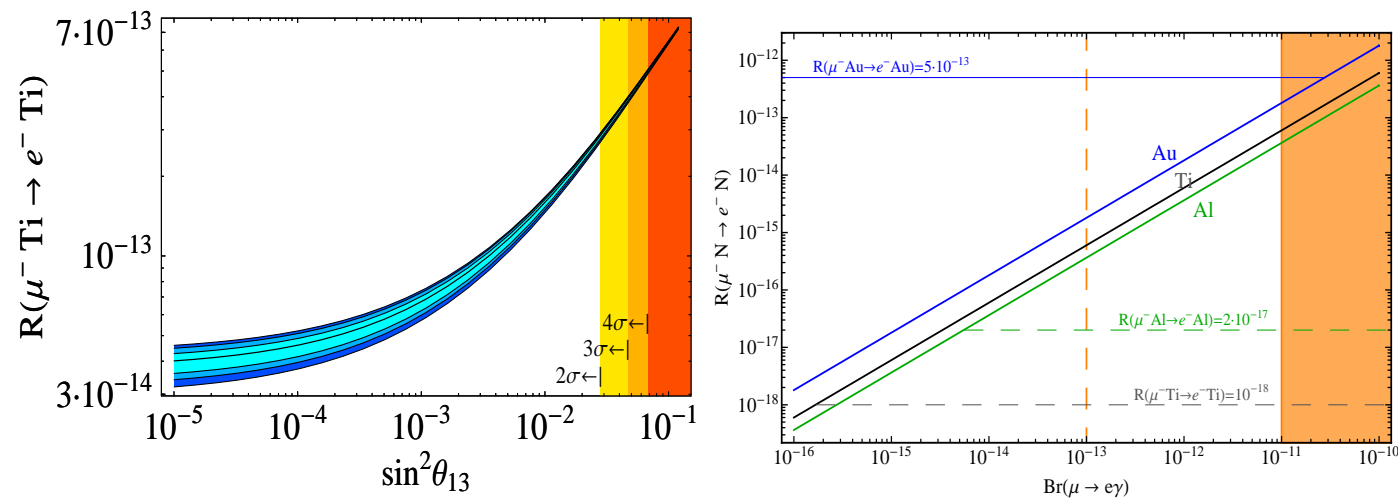

FIGURE 6. Inverse seesaw model expectations for $\mu \rightarrow e \gamma$ versus $\sin ^{2} \theta_{13}$ (left panel), and correlation of $\mu \rightarrow e \gamma$ with $\mu^{-}-e^{-}$conversion rates (right panel), details in Ref. [19].

\section{How do neutrinos get mass?}

First note that charged fermions in the Standard Model (SM) come in two chiral species so that they acquire mass when the electroweak symmetry breaks through the nonzero vacuum expectation value (vev) of the Higgs scalar doublet $\langle\Phi\rangle$. Neutrinos do not. There is, however, an effective lepton number violating dimension-five operator $\lambda L \Phi L \Phi$ in Fig. 7, which can be added to the SM (here $L$ denotes any of the lepton doublets) [59]. This induces Majorana neutrino masses quadratic in the Higgs vev $\langle\Phi\rangle$
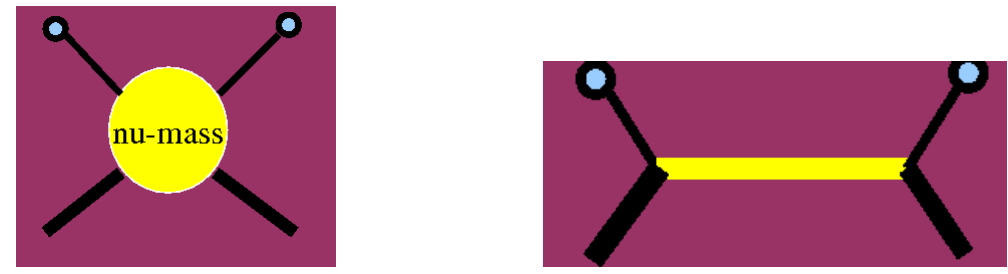

FIGURE 7. Dimension-5 operator responsible for neutrino mass [59] and its type-I seesaw realization.

(indicated by small blobs in Fig. 7), in contrast to the linear behavior of the charged fermion masses. This would provide a natural way to account for the smallness of neutrino masses, irrespective of their specific origin. Little more can be said from first principles about the mechanism giving rise to this operator, its associated mass scale or its flavour structure. Its strength $\lambda$ may be suppressed by a large scale $M_{X}$ in the denominator (top-down) scenario, leading to $m_{v}=\lambda_{0} \frac{\langle\Phi\rangle^{2}}{M_{X}}$, where $\lambda_{0}$ is some unknown dimensionless constant.

Since gravity has been argued to break global symmetries, it could induce the dimension-five operator, with $M_{X}=M_{P}$, the Planck scale [61]. However in this case the resulting Majorana neutrino masses are too small, and hence one needs physics beyond the Standard Model to account for current data.

A popular way to to generate the dimension-5 operator by the exchange of heavy states, typically fermions (type-I seesaw) as illustrated in Fig. 7 right. However, also heavy scalars (type-II seesaw) can do the job, as shown in Fig. 8, The so-called type-I 


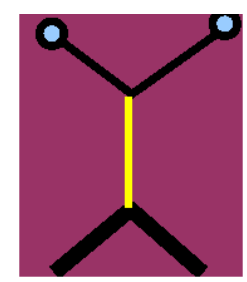

FIGURE 8. Type-II seesaw mechanism [3].

seesaw mechanism was first mentioned in Ref. [62] while the type-II seesaw mechanism was first mentioned in Ref. [3] as part of the general classification of neutrino massgiving schemes. The hierarchy of vevs was discussed in Ref. [63], along with the detailed perturbative seesaw diagonalization method. The main point is that, as the masses of the intermediate states go to infinity, neutrinos become light. The seesaw provides a simple realization of Weinberg's dimension-5 operator [59]. It can be implemented in many ways, with explicitly or spontaneously broken B-L, gauged or not; with different gauge groups and multiplet contents, minimal or not; with its basic scale large or small. All of this, the original references, together with many other variants of the seesaw mechanism [18, 64, 65, 66] are reviewed in [38, 7]. Detailed model-independent aspects of seesaw phenomenology, e. g. the structure of its lepton mixing matrix are given in [3].

Alternatively, $\lambda$ could vanish due to symmetry [60] or be suppressed by small parameters (e.g. scales, Yukawa couplings) and/or loop-factors (bottom-up scenario) with no need for a large scale, opening the door to new processes associated with the new states required to provide the neutrino mass and which could be searched for, e. g., at the LHC. This is the case in radiative schemes, where neutrino masses arise as calculable loop corrections [21, 22] as illustrated in Fig. 9. The field $\sigma$ in the right panel is an $S U(3) \otimes S U(2) \otimes U(1)$ singlet whose vev breaks lepton number and induces the neutrino masses, as in Ref. [67]. Clearly, in this case neutrino masses are suppressed by a product of three small Yukawas, two charged lepton masses, in addition to the two-loop factor.

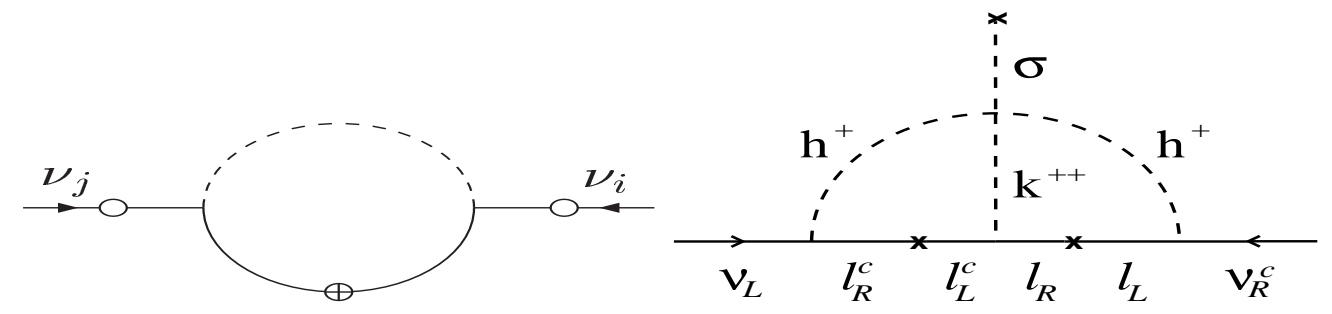

FIGURE 9. Radiative origin for neutrino mass.

An example of hybrid neutrino masses is provided by supersymmetry, which gives a plausible and experimentally testable origin for neutrino mass. Indeed, the intrinsically supersymmetric way to break lepton number is to break the so-called $\mathrm{R}$ parity. This may happen spontaneously, driven by a nonzero vev of an $S U(3) \otimes S U(2) \otimes U(1)$ singlet sneutrino [68, 69, 70], leading to an effective model with bilinear violation of $\mathrm{R}$ parity [71, 72]. This provides the minimal way to add neutrino masses to the MSSM [72]. 
Neutrino mass generation is hybrid, with typically a normal hierarchy mass spectrum where one scale (atmospheric) is generated at tree level through a weak-scale seesaw and the other (solar) is induced by calculable one-loop corrections. This is illustrated in the left panel of Fig. 9, where open blobs denote the $\Delta L=1$ insertions present in the R-parity MSSM [73].

Such low-scale models of neutrino mass offer the tantalizing possibility of reconstructing neutrino mixing at high energy accelerators, like the LHC and the ILC. A clear example is provided by models where supersymmetry is the origin of neutrino mass. A general feature of these models is that, unprotected by any symmetry, the lightest supersymmetric particle (LSP) is expected to decay inside the detector [73] [74]. More strikingly, its decay properties correlate with the neutrino mixing angles. For example, if the LSP is the lightest neutralino, it should have the same decay rate into muons and taus, since the observed atmospheric angle is close to $\pi / 4$ [75, 76, 77]. Such correlations hold irrespective of which supersymmetric particle is the lightest [78] and constitute a smoking gun signature of this proposal that will be tested at upcoming accelerators.

\section{How to understand mixing angles?}

Current neutrino oscillation data indicate the existence of two large lepton mixing angles, while quark mixing angles are all small. This is rather difficult to explain from first principles in unified schemes where quarks and leptons are related. Phenomenologically, there seems to be an intriguing complementarity between quark and lepton mixing angles [79, 80, 81, 82]. There have been many attempts to understand the values of the leptonic mixing angles from underlying symmetries, a major challenge facing model-builders.

Harrison, Perkins \& Scott noted [83] that the neutrino mixing angles are approximately given by,

$$
\begin{aligned}
\tan ^{2} \theta_{\mathrm{ATM}} & =\tan ^{2} \theta_{23}^{0}=1 \\
\sin ^{2} \theta_{\mathrm{Chooz}} & =\sin ^{2} \theta_{13}^{0}=0 \\
\tan ^{2} \theta_{\mathrm{SOL}} & =\tan ^{2} \theta_{12}^{0}=0.5 .
\end{aligned}
$$

Such pattern could result from some flavour symmetry valid at high energy scales. Its predictions should then be corrected by renormalization group evolution [84, 85, 86].

Here I consider a specific idea to predict neutrino masses and mixing angles: that neutrino masses arise from a common seed at some "neutrino mass unification" scale $M_{X}$ [87], very similar to the merging of the SM gauge coupling constants at high energies due to supersymmetry [88]. Although in its simplest form this idea is now inconsistent (at least if CP is conserved) with the observed value of the solar mixing angle $\theta_{12}$, there is an alternative realization in terms of an $A_{4}$ flavour symmetry which is both viable and predictive [39]. Starting from three-fold degeneracy of the neutrino masses at the seesaw scale, the model predicts maximal atmospheric angle and vanishing $\theta_{13}$,

$$
\theta_{23}=\pi / 4 \text { and } \theta_{13}=0
$$


Although the solar angle $\theta_{12}$ is unpredicted, one expects 3

$$
\theta_{12}=\mathscr{O}(1)
$$

If $\mathrm{CP}$ is violated $\theta_{13}$ becomes arbitrary and the Dirac phase is maximal [89]. One can show that lepton and slepton mixings are closely related and that there must exist at least one slepton below $200 \mathrm{GeV}$, which can be produced at the LHC. The absolute Majorana neutrino mass scale $m_{0} \geq 0.3 \mathrm{eV}$ ensures that the model will be probed by future cosmological tests and $\beta \beta_{0 v}$ searches. Rates for lepton flavour violating processes $l_{j} \rightarrow t_{i}+\gamma$ typically lie in the range of sensitivity of coming experiments, with $\mathrm{BR}(\mu \rightarrow e \gamma) \gtrsim 10^{-15}$ and $\mathrm{BR}(\tau \rightarrow \mu \gamma)>10^{-9}$.

\section{NEUTRINOS AS COSMOLOGICAL PROBES}

Optical telescopes can only probe the recent epochs of the evolution of the Universe, after their last scattering surface, about $400.000 \mathrm{yr}$ after the primordial Bang. In contrast neutrinos can probe much earlier stages in the evolution of the early Universe, such as nucleosynthesis, and even earlier periods. Indeed, if neutrino masses are generated at high scale, early enough in the evolution of the Universe, a la seesaw, previous to the electroweak phase transition, they may provide the seed both for the observed baryon asymmetry of the Universe, and the dark matter, as I now discuss.

\section{Thermal leptogenesis}

Seesaw models open an attractive possibility of accounting for the observed cosmological matter-antimatter asymmetry in the Universe through leptogenesis [90]. In this picture the decays of the heavy "right-handed" neutrinos present in the seesaw take place before the electroweak phase transition [91] through diagrams in Fig. 10. They may also violate $\mathrm{CP}$ with rates smaller than the Hubble expansion rate at that epoch. Under these circumstances, the lepton (or B-L) asymmetry thus produced gets con-

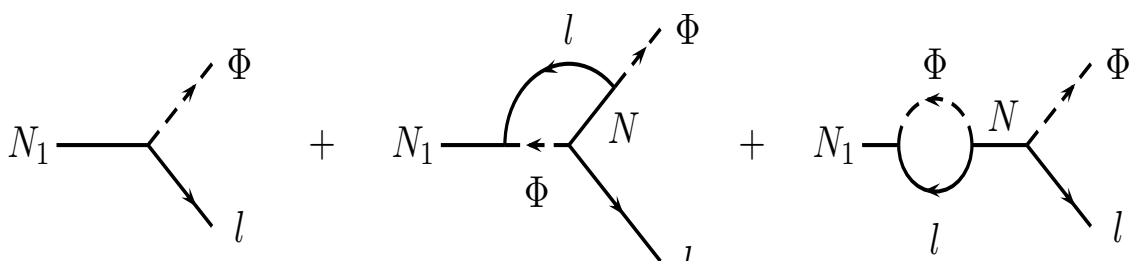

FIGURE 10. Diagrams contributing to leptogenesis.

verted, through sphaleron processes, into the observed baryon asymmetry. However, in typical supersymmetric seesaw schemes the high temperature needed for leptogenesis leads to an overproduction of gravitinos, which destroys the standard Big Bang Nu-

\footnotetext{
${ }^{3}$ There have been realizations of the $A_{4}$ symmetry that also predict the solar angle, e. g. Ref. [42].
} 
cleosynthesis $(\mathrm{BBN})$ predictions. This happens in minimal supergravity models, with $m_{3 / 2} \sim 100 \mathrm{GeV}$ to $10 \mathrm{TeV}$, where gravitinos decay during or after BBN. To prevent such gravitino crisis one requires an upper bound on the reheat temperature $T_{R}$ after inflation, since the abundance of gravitinos is proportional to $T_{R}$. This leads to a stringent upper bound [92], which is in conflict with the temperature required for leptogenesis, $T_{R}>2 \times 10^{9} \mathrm{GeV}[93]$. One way to cure this conflict [94] is to add a small R-parity violating $\lambda_{i} \hat{v}^{c}{ }_{i} \hat{H}_{u} \hat{H}_{d}$ term in the superpotential, where $\hat{v}^{c}{ }_{i}$ are right-handed neutrino supermultiplets. One can show that in the presence of this term, the produced leptonantilepton asymmetry can be enhanced. An alternative suggestion [95] was made in the context of extended $\mathrm{SO}(10)$ supersymmetric seesaw schemes. It was shown in this case that leptogenesis can occur at relatively low scales, TeV or so, through the decay of a new singlet, thereby avoiding the gravitino crisis. Washout of the asymmetry is effectively suppressed by the absence of direct couplings of the singlet to leptons. The presence of extra chiral singlets also helps to reconcile the large lepton mixing angles with small quark mixing angles, within the framework of the successful Fritzsch ansatz. As illustrated in Fig. 11 sizeable asymmetry can be generated just from the leptonic CP violation parameter $\delta$ that characterizes neutrino oscillations.

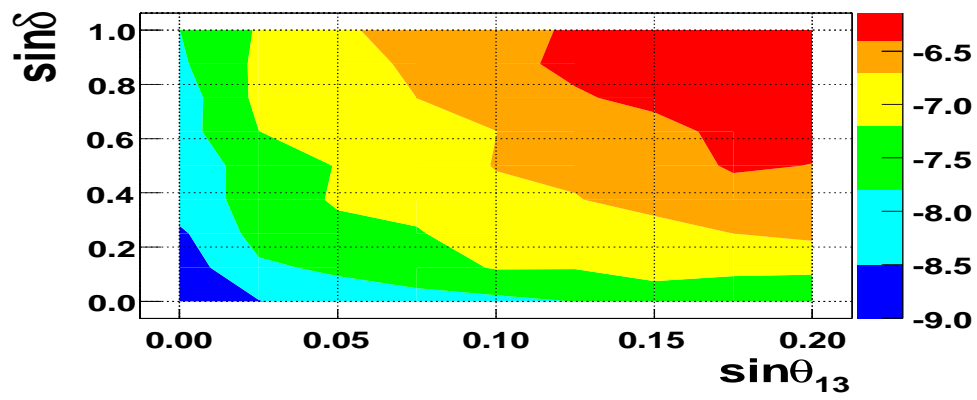

FIGURE 11. Sizeable leptogenesis in supersymmetric $\mathrm{SO}(10)$ models, from [96].

\section{Neutrino masses and dark matter}

An attractive way to generate neutrino masses as required to account for current neutrino oscillation data involves the spontaneous breaking of lepton number. Due to quantum gravity effects the associated Goldstone boson - the majoron - is likely to pick up a mass. If its mass lies in the kilovolt scale, the majoron can play the role of late-decaying Dark Matter, decaying mainly to neutrinos. However, cosmic microwave background observations place constraints [97], on the majoron lifetime and mass, illustrated in Fig. 12, Such majoron decaying dark matter scenario fits nicely in models where neutrino masses arise a la seesaw, where the majoron couples to photons due to the presence of a Higgs triplet. In this case it may be tested as it has also a sub-dominant decay to two photons leading to a mono-energetic emission line. Comparison of expected photon emission rates with observations leads to model independent restrictions on the relevant parameters, illustrated in Fig. 13 the resulting 

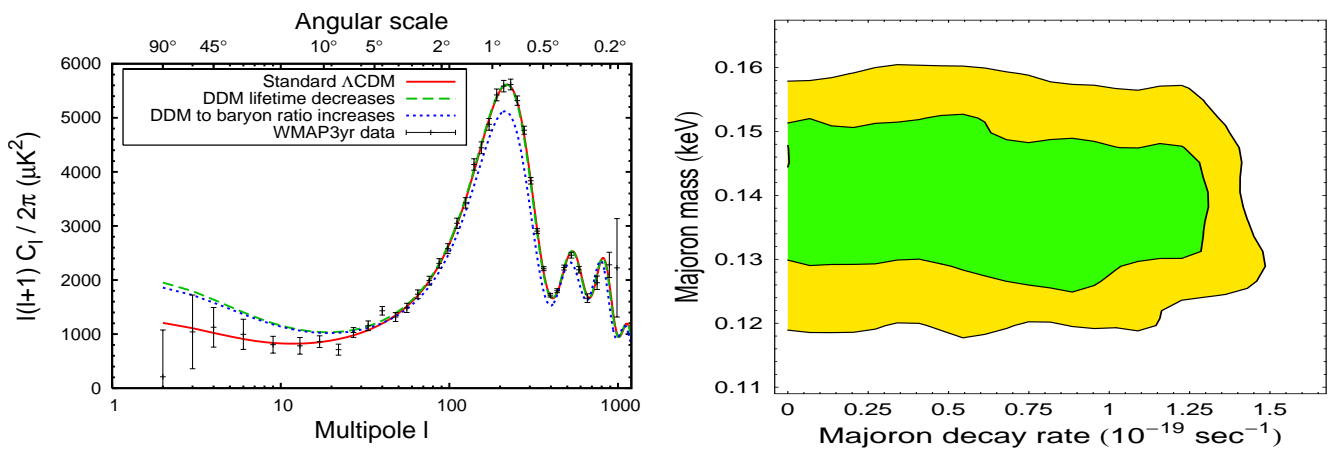

FIGURE 12. Late decaying majoron dark matter parameters, from [97].
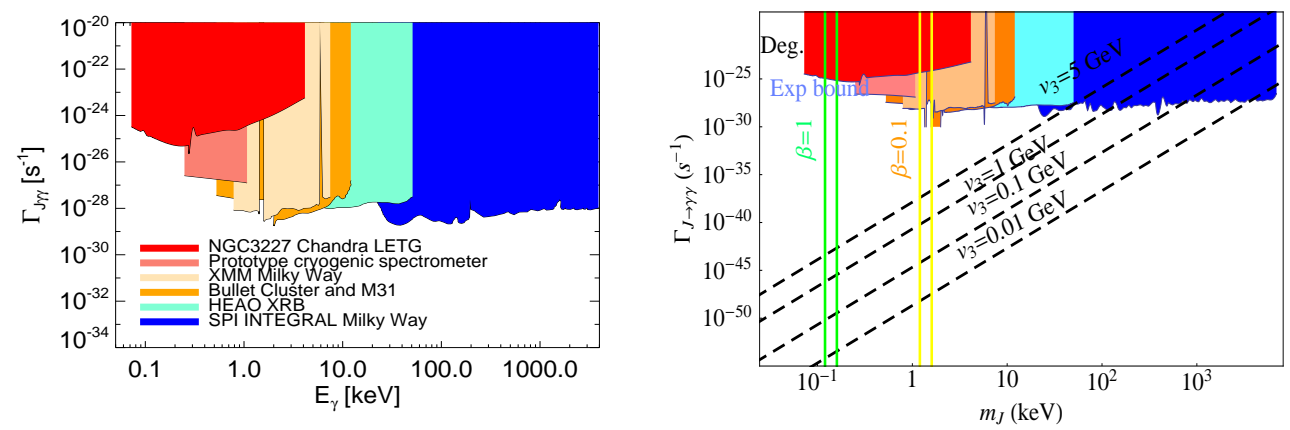

FIGURE 13. Testing the majoron late decaying dark matter, from [98].

sensitivities within an explicit seesaw realization,

Finally, I also mention that neutrino mass may open new possibilities for "conventional" supersymmetric dark matter, widely discussed here. It can be shown [99] that, within the inverse seesaw mechanism for generating neutrino masses [18] minimal supergravity is more likely to have a sneutrino as the lightest superparticle than the conventional neutralino. Such schemes naturally reconcile the small neutrino masses with the correct relic sneutrino dark matter abundance and accessible direct detection rates in nuclear recoil experiments [99].

\section{Acknowledgments:}

I thank the organizers for hospitality and Martin Hirsch for reading the manuscript. This work was supported by Spanish grants FPA2008-00319/FPA2005-01269 and FPA2008-01935-E/FPA, as well as European commission RTN Contract MRTN-CT2004-503369 and ILIAS/N6 Contract RII3-CT-2004-506222.

\section{REFERENCES}

1. Neutrino2008 Web-page http://www2.phys.canterbury.ac.nz/ jaa53/and talks given therein.

2. Schwetz, T., Tortola, M. A., and Valle, J. W. F., arXiv:0808.2016 [hep-ph]; for analysis details and the relevant experimental references see review by Maltoni, M., et al., New J. Phys., 6, 122 (2004). 
3. Schechter, J., and Valle, J. W. F., Phys. Rev., D22, 2227 (1980).

4. Schechter, J., and Valle, J. W. F., Phys. Rev., D23, 1666 (1981). Bilenky, S. M., Hosek, J., and Petcov, S. T., Phys. Lett., B94, 49 (1980). Doi, M., Kotani, T., Nishiura, H., Okuda, K., and Takasugi, E., Phys. Lett., B102, 323 (1981).

5. $\quad$ Schechter, J., and Valle, J. W. F., Phys. Rev., D21, 309 (1980).

6. Bandyopadhyay, A. et al. [ISS Physics Working Group], arXiv:0710.4947 [hep-ph].

7. Nunokawa, H., Parke, S. J., and Valle, J. W. F., Prog. Part. Nucl. Phys. 60 (2008) 338 [arXiv:0710.0554[hep-ph]].

8. $\quad$ Burgess, C. P., et al., JCAP, 0401, 007 (2004).

9. Burgess, C., et al., W. F., Mon. Not. Roy. Astron. Soc., 348, 609 (2004).

10. Burgess, C., et al., Astrophys. J., 588, L65 (2003).

11. Miranda, O. G., et al., Nucl. Phys., B595, 360-380 (2001).

12. Guzzo, M., et al., Nucl. Phys., B629, 479-490 (2002).

13. Barranco, J., et al., Phys. Rev., D66, 093009 (2002).

14. Miranda, O. G., Rashba, T. I., Rez, A. I., and Valle, J. W. F., Phys. Rev., D70, 113002 (2004).

15. Pakvasa, S., and Valle, J. W. F. (2003), proc. of the Indian National Acad. of Sciences on Neutrinos, Vol. 70A, No.1, p.189 - 222 (2004), Eds. Indumathi, Murthy \& Rajasekaran. [hep-ph/0301061]

16. Gonzalez-Garcia, M. et al., Phys. Rev., D63, 033005 (2001).

17. Valle, J. W. F., Phys. Lett., B199, 432 (1987).

18. Mohapatra, R. N., and Valle, J. W. F., Phys. Rev., D34, 1642 (1986).

19. Deppisch, F., and Valle, J. W. F., Phys. Rev., D72, 036001 (2005);

Deppisch, F., Kosmas, T. S., and Valle, J. W. F. Nucl. Phys. B752, 80 (2006).

20. Hall, L. J., Kostelecky, V. A., and Raby, S., Nucl. Phys., B267, 415 (1986).

21. Zee, A., Phys. Lett., B93, 389 (1980).

22. Babu, K. S., Phys. Lett., B203, 132 (1988).

23. Gonzalez-Garcia, M. et al., Phys. Rev. Lett. 82 (1999) 3202 [arXiv:hep-ph/9809531].

24. Fornengo, N., et al., Phys. Rev., D65, 013010 (2002).

25. Huber, P., and Valle, J. W. F., Phys. Lett., B523, 151-160 (2001).

26. Miranda, O. G., Tortola, M. A., and Valle, J. W. F., JHEP, 10, 008 (2006).

27. Huber, P., Schwetz, T., and Valle, J. W. F., Phys. Rev. Lett., 88, 101804 (2002);

Phys. Rev. D66, 013006 (2002)

28. Schechter, J., and Valle, J. W. F., Phys. Rev., D25, 2951 (1982).

29. Schechter, J., and Valle, J. W. F., Phys. Rev., D24, 1883 (1981), err. D25, 283 (1982).

30. Wolfenstein, L., Phys. Lett., B107, 77 (1981).

31. Pal, P. B., and Wolfenstein, L., Phys. Rev., D25, 766 (1982).

32. Kayser, B., Phys. Rev., D26, 1662 (1982).

33. Avignone, F., Elliott, S., and Engel, J., Rev. Mod. Phys. 80 (2008) 481 [arXiv:0708.1033 [nucl-ex]].

34. Hirsch, M., Kovalenko, S., and Schmidt, I., Phys. Lett., B642, 106 (2006).

35. Drexlin, G., Nucl. Phys. Proc. Suppl., 145, 263-267 (2005).

36. Lesgourgues, J., and Pastor, S., Phys. Rep., 429, 307-379 (2006); Hannestad, S. hep-ph/0602058 Fogli, G. L., et al., Phys. Rev., D70, 113003 (2004).

37. V. A. Rodin, A. Faessler, F. Simkovic and P. Vogel, Nucl. Phys. A 766 (2006) 107 [Erratum-ibid. A 793 (2007) 213] [arXiv:0706.4304 [nucl-th]].

38. Valle, J. W. F. Corfu lectures, Sept 2005, J. Phys. Conf. Ser. 53 (2006) 473 [arXiv:hep-ph/0608101].

39. Babu, K. S., Ma, E., and Valle, J. W. F., Phys. Lett., B552, 207 (2003);

M. Hirsch, et al. Phys. Rev. D 69 (2004) 093006 [arXiv:hep-ph/0312265].

40. Caldwell, D. O., and Mohapatra, R. N., Phys. Rev., D48, 3259 (1993).

41. Ioannisian, A., and Valle, J. W. F., Phys. Lett., B332, 93 (1994).

42. Hirsch, M., et. al., Phys. Rev., D72, 091301 (2005), Err. D72, 119904 (2005).

43. Klapdor-Kleingrothaus, H. V., et al., Phys. Lett., B586, 198-212 (2004).

44. Aalseth, C. E., et al., Mod. Phys. Lett., A17, 1475-1478 (2002).

45. Aalseth, C. E., et al., Phys. Rev., D65, 092007 (2002).

46. Saakyan, R., Nones, C., Tomei, C., and Zuber, K. talk at ILIAS/N6 WG1 Collaboration meeting, DBD06, April 28 \& 29 (2006), Valencia.

47. Bernabeu, J., et al., Phys. Lett., B187, 303 (1987).

48. Gonzalez-Garcia, M. C., and Valle, J. W. F., Mod. Phys. Lett., A7, 477-488 (1992). 
49. Ilakovac, A., and Pilaftsis, A., Nucl. Phys., B437, 491 (1995).

50. Hall, L. J., Kostelecky, V. A., and Raby, S., Nucl. Phys., B267, 415 (1986).

51. Borzumati, F., and Masiero, A., Phys. Rev. Lett., 57, 961 (1986).

52. Casas, J. A., and Ibarra, A., Nucl. Phys., B618, 171-204 (2001).

53. Antusch, S., Arganda, E., Herrero, M. J., and Teixeira, A. hep-ph/0607263.

54. Hirsch, M. et al, Phys. Rev. D78, 013006 (2008), arXiv:0804.4072[ [hep-ph].

55. Branco, G. C., Rebelo, M. N., and Valle, J. W. F., Phys. Lett., B225, 385 (1989).

56. Rius, N., and Valle, J. W. F., Phys. Lett., B246, 249-255 (1990).

57. Kuno, Y., AIP Conf. Proc., 542, 220-225 (2000).

58. Dittmar, M., et al., Nucl. Phys., B332, 1 (1990); P. Abreu et al., Z. Phys. C74, 57 (1997).

59. Weinberg, S., Phys. Rev., D22, 1694 (1980).

60. Gogoladze, I., Okada, N. and Shafi, Q., arXiv:0809.0703 [hep-ph].

61. de Gouvea, A., and Valle, J. W. F., Phys. Lett., B501, 115-127 (2001).

62. Minkowski, P., Phys. Lett., B67, 421 (1977).

63. Schechter, J., and Valle, J. W. F., Phys. Rev., D25, 774 (1982).

64. Gonzalez-Garcia, M. C., and Valle, J. W. F., Phys. Lett., B216, 360 (1989).

65. Akhmedov, E., et al., Phys. Rev., D53, 2752-2780 (1996); Phys. Lett. B368, 270 (1996)

66. Malinsky, M., Romao, J. C., and Valle, J. W. F., Phys. Rev. Lett., 95, 161801 (2005).

67. Peltoniemi, J. T., and Valle, J. W. F., Phys. Lett., B304, 147-151 (1993).

68. Masiero, A., and Valle, J. W. F., Phys. Lett., B251, 273-278 (1990).

69. Romao, J. C., Santos, C. A., and Valle, J. W. F., Phys. Lett., B288, 311-320 (1992).

70. Romao, J. C., Ioannisian, A., and Valle, J. W. F., Phys. Rev., D55, 427-430 (1997).

71. Diaz, M. A., Romao, J. C., and Valle, J. W. F., Nucl. Phys., B524, 23-40 (1998).

72. Hirsch, M., and Valle, J. W. F., New J. Phys., 6, 76 (2004).

73. Hirsch, M., et al., Phys. Rev., D62, 113008 (2000), [err-ibid. D65 119901 (2002)];

Diaz, M. et al., Phys. Rev. D 68013009 (2003), [Err-ibid. D71 059904 (2005)]

74. de Campos, F., et al., Phys. Rev., D71, 075001 (2005).

75. Porod, W., Hirsch, M., Romao, J., and Valle, J. W. F., Phys. Rev., D63, 115004 (2001).

76. Romao, J. C., et al., Phys. Rev., D61, 071703 (2000).

77. Mukhopadhyaya, B., Roy, S., and Vissani, F., Phys. Lett., B443, 191-195 (1998).

78. Hirsch, M., and Porod, W., Phys. Rev., D68, 115007 (2003).

79. Raidal, M., Phys. Rev. Lett., 93, 161801 (2004).

80. Minakata, H., and Smirnov, A. Y., Phy. Rev., D70, 073009 (2004).

81. Ferrandis, J., and Pakvasa, S., Phys. Rev., D71, 033004 (2005).

82. Dighe, A., Goswami, S., and Roy, P., Phys. Rev., D73, 071301 (2006).

83. Harrison, P. F., and Scott, W. G., Phys. Lett., B535, 163-169 (2002),

Harrison, P. F., Perkins, D. H., and Scott, W. G., Phys. Lett., B530, 167 (2002).

84. Altarelli, G., and Feruglio, F., Nucl. Phys., B720, 64-88 (2005).

85. Hirsch, M., et al. Phys. Rev. D 75 (2007) 053006 [arXiv:hep-ph/0606082].

86. Altarelli, G., and Feruglio, F., New J. Phys., 6, 106 (2004).

87. Chankowski, P., Ioannisian, A., Pokorski, S., and Valle, J. W. F., Phys. Rev. Lett., 86, 3488 (2001).

88. Amaldi, U., de Boer, W., and Furstenau, H., Phys. Lett., B260, 447-455 (1991).

89. Grimus, W., and Lavoura, L., Phys. Lett., B579, 113-122 (2004).

90. Fukugita, M., and Yanagida, T., Phys. Lett., B174, 45 (1986).

91. Kuzmin, V. A., Rubakov, V. A., and Shaposhnikov, M. E., Phys. Lett., B155, 36 (1985).

92. Kawasaki, M., Kohri, K., and Moroi, T., Phys. Rev., D71, 083502 (2005).

93. Buchmuller, W., Di Bari, P., and Plumacher, M. Annals Phys. 315, 305 (2005)

94. Farzan, Y., and Valle, J. W. F., Phys. Rev. Lett., 96, 011601 (2006).

95. M. Hirsch, et al. Phys. Rev. D 75 (2007) 011701 [arXiv:hep-ph/0608006].

96. Romao, J. C., et. al., Phys. Rev. D 77 (2008) 055002 [arXiv:0707.2942 [hep-ph]].

97. Lattanzi, M. and Valle, J. W. F., Phys. Rev. Lett. 99 (2007) 121301 [arXiv:0705.2406 [astro-ph]].

98. Bazzocchi, F. et al, JCAP 0808 (2008) 013 [arXiv:0805.2372 [astro-ph]].

99. Arina, C., et al. Phys. Rev. Lett. 101 (2008) 161802, arXiv:0806.3225 [hep-ph];

Cerdeno, D., Munoz, C. and Seto, O., arXiv:0807.3029 [hep-ph], and talks by Arina and Cerdeno. 